\title{
Targeting $M Y C$-inducing enhancer-associated noncoding (MYC-IEANC) RNAs inhibits the proliferation of HCC cells
}

Hae In Choi

Hanyang University - Seoul Campus: Hanyang University

Jin Choul Chai

Seoul National University College of Veterinary Medicine

Young Seek Lee

Seoul National University College of Veterinary Medicine

Kyoung Hwa Jung

Korea Polytechnics II Incheon Campus

Young Gyu Chai ( $\nabla$ ygchai@hanyang.ac.kr)

Hanyang University - ERICA Campus: Hanyang University - Ansan Campus https://orcid.org/00000002-3333-4803

Research

Keywords: Enhancer, MYC, eRNA, ASO, HCC

Posted Date: August 6th, 2021

DOl: https://doi.org/10.21203/rs.3.rs-754221/v1

License: (c) (i) This work is licensed under a Creative Commons Attribution 4.0 International License.

Read Full License 


\section{Abstract \\ Background}

MYC, a critical oncogene, encodes the c-MYC transcription factor (TF) and plays an essential role in hepatocellular carcinoma (HCC) development. Recent studies have identified numerous tissue-specific enhancers of $M Y C$ in various cancers, but an HCC-specific enhancer of $M Y C$ remains elusive.

\section{Methods}

We analyzed enhancer markers, including H3K27ac enrichment and enhancer RNA (eRNA) expression, to determine putative enhancer regions of MYC in HCC cells. Enhancer activity was detected using a dualluciferase reporter assay. We used the CRISPR-Cas9 system to edit the enhancer regions and performed antisense oligonucleotide (ASO) to inhibit eRNA. The functions of enhancers and eRNAs on HCC cells were confirmed by cell proliferation assay and sphere formation assay.

\section{Results}

We choose two active enhancers R2 and R3, with high activity among six putative enhancer regions by analyzing enhancer markers. Enhancer R2 and R3 are present approximately $800 \mathrm{~kb}$ downstream from the $M Y C$ gene. We confirmed eRNA activities in the enhancer regions. Depletion of these enhancer regions inhibited eRNAs significantly reduced MYC expression. In addition, MYC enhancers and eRNAs regulated HCC cell proliferation and progression.

\section{Conclusion}

In this study, we present $M Y C$ enhancers in $\mathrm{HCC}$ and elucidate the molecular functions of $M Y C$-inducing enhancer-associated noncoding (MYC-IEANC) RNAs in the proliferation of HCC cells. Furthermore, our results suggest that $M Y C$-IEANC RNAs, which play an oncogenic role in HCC cells, can be a target for HCC treatment.

\section{Background}

Enhancers, as epigenetic regulators, are short genomic elements that provide specificity to target gene expression. Enhancer activity is regulated by various factors associated with epigenetic modification [1]. Therefore, the activity and location of enhancers are known to be cell type-specific and are bound by tissue- or cell type-specific transcription factors (TFs) [2]. Enhancers consist of DNA enriched with $\mathrm{H} 3 \mathrm{~K} 27 \mathrm{ac}$ and H3K4me marks and are closely related to critical oncogenic drivers [3]. Accordingly, studies have been actively conducted to elucidate the regulation of cancer by identifying and regulating enhancers that function differently depending on the cancer type [4-6]. Perturbing enhancer activity has 
revolutionized cancer treatment $[7,8]$. These therapies have been developed based on the ability of cancers to undergo aberrant transcription through the dysregulation of enhancers.

eRNA, a noncoding transcript generated by active enhancers, plays an essential role in regulating gene expression along with enhancers [9]. eRNAs are expressed cell type-specific to control cell fate, and in cancer, they can be used as new cancer diagnostic markers and drugs [10,11]. There are many studies on various methods for eRNA control and their effects; among them, inhibition of eRNA and functional regulation using antisense oligonucleotide (ASO) has significant results that can be utilized to treat various diseases including cancer [12-14].

MYC is essential for various cellular processes, including cell growth, proliferation, differentiation, and apoptosis [15]. MYC acts as an oncogene and tightly regulates the normal state. In contrast, dysregulation of $M Y C$ is prevalent in cancer. And upregulation of $M Y C$ expression is observed in $50-60 \%$ of all cancers $[16,17]$. Accordingly, studies on the enhancer of $M Y C$ were also conducted, and it was found that the location of enhancers is cancer-specific [18]. As a representative example, the enhancer is located at approximately $0.7 \mathrm{Mb}$ downstream of $M Y C$ in prostate cancer and relatively close to $70 \mathrm{~kb}$ upstream in pancreatic cancer. In contrast, it is located 1.9 Mb upstream of $M Y C$ in glioma and regulates MYC expression [19-21]. However, studies on MYC enhancers in liver cancer have been insufficient.

In this study, we identified regions presumed to be enhancers of $M Y C$ that regulate oncogene $M Y C$ expression. We confirmed the activity of eRNA as well as the enhancer. In addition, this study suggests that ASO may be a therapeutic agent by efficiently decreasing enhancer activity and inhibiting eRNA expression, resulting in the inhibition of cell proliferation and sphere formation in HCC.

\section{Methods}

\section{Cell culture of HCCs}

The HepG2 and Huh7 HCC cell lines were purchased from Korean Cell Line Bank (KCLB) and maintained in minimum essential medium or RPMI-1640 medium supplemented with $10 \%$ fetal bovine serum (FBS) and penicillin (100 units $/ \mathrm{ml}) /$ streptomycin $(100 \mathrm{mg} / \mathrm{ml})$ (Thermo Fisher Scientific). The medium was replaced every 3-4 days. The cells were maintained in a humidified incubator with $95 \%$ air and a $5 \% \mathrm{CO}_{2}$ atmosphere at $37^{\circ} \mathrm{C}$. JQ1, OTX-015, C646, and 5,6-dichlorobenzimidazole 1- $\beta$-D-ribofuranoside (DRB) were purchased from Tocris Bioscience (Minneapolis). JQ1, OTX-015, C646, and DRB were dissolved in dimethyl sulfoxide (DMSO) at a stock concentration of $10 \mathrm{mM}$. The cells were treated with different concentrations of JQ1 and DRB for different durations.

\section{Cell proliferation assay (WST-1 assay)}

The cell proliferation assay was performed using a premixed water-soluble tetrazolium salt (WST-1) cell viability test (Takara) according to the manufacturer's instructions. The cells were seeded at a density of $1 \times 10^{4}$ cells per well. WST-1 was added to each well, and the absorbance of the microplate at 450 
$\mathrm{nm}$ was measured after an additional $4 \mathrm{~h}$ incubation. The data represent three independent experiments $(n=3)$.

Ethynyl deoxyuridine (EdU) analysis was performed using an EdU cell proliferation assay kit (Invitrogen) following the manufacturer's instructions. Then, the cells were washed with phosphate-buffered saline, mounted with a 4',6-diamidino-2-phenylindole (DAPI)-containing mounting solution (VECTASHIELD, Vector Laboratories), and imaged by microscopy (Nikon Eclipse 80i). The percentage of EdU-positive cells was examined in HCC cell lines using ImageJ (Bethesda) software. The data represent three independent experiments $(n=3)$.

\section{Gene expression analysis using quantitative PCR (qRT-PCR)}

Total RNA was extracted from HepG2 cells using RNAiso Plus (Takara) according to the manufacturer's instructions. cDNA was synthesized by PrimeScript reverse transcriptase (Takara) and amplified using gene-specific primers. The primers used for qRT-PCR are listed in Additional file 1: Table 1. The primers were designed by Primer Bank (https://pga.mgh.harvard.edu/primerbank/). qRT-PCR was performed with TBGreen Premix Ex Taq II (Takara). Glyceraldehyde-3-phosphate dehydrogenase (GAPDH) was used as an internal control. The data represent three independent experiments $(n=3)$. After performing qRT-PCR, the results were analyzed using the critical threshold $\left(\triangle C_{T}\right)$ and the comparative critical threshold $\left(\triangle \triangle \mathrm{C}_{\mathrm{T}}\right.$ ) methods in $\mathrm{ABI} 7500$ (Applied Biosystems) software with the NormFinder and geNorm PLUS algorithms.

\section{Luciferase reporter assay}

Enhancer regions (R1 R6) were amplified using LongAmp Taq 2X Master Mix (New England Biolabs). Enhancer regions were amplified using forward and reverse primers to generate Nhel of Sad and Xhol sites, respectively. These constructs were cloned into the pGL4.26 construct (Promega). The primers used for cloning are listed in Additional file 1: Table 2. The cells were seeded into 24-well plates and transfected with Lipofectamine 3000 (Thermo Fisher Scientific). According to the manufacturer's instructions, luciferase activity was measured using the Dual-Glo Luciferase Assay kit (Promega). PRL-TK (Renilla luciferase expression construct; Promega) was used as an internal control. Luciferase activity was normalized to Renilla luciferase and the control (empty vector).

\section{Construction of R3 region KO HCCs using the CRISPR-Cas9 system}

Forward and reverse oligomers for gRNA synthesis against target sites were designed according to the manufacturer's instructions. The oligomers were extended into 100-mer insert DNA using Phusion HighFidelity PCR Master Mix (M0531, Biolabs) with the following setup: 2 min at $98^{\circ} \mathrm{C}, 4$ cycles of amplification ( $10 \mathrm{sec}$ at $98^{\circ} \mathrm{C}, 20 \mathrm{sec}$ at $53^{\circ} \mathrm{C}, 30 \mathrm{sec}$ at $72^{\circ} \mathrm{C}$ ), and $5 \mathrm{~min}$ at $72^{\circ} \mathrm{C}$. Then, the insert DNA was purified and combined with gRNA_Cloning Vector (\#41824, Addgene) using Gibson Assembly Master Mix (E2611, New England Biolabs) at $50^{\circ} \mathrm{C}$ for $1 \mathrm{~h}$, followed by transformation and colony PCR. The cloned vectors were then purified and ordered to be sequenced (Macrogen) to confirm the recombination. 
HCCs were transfected with the recombinant gRNA plasmid vector and pCas9_GFP plasmid (\#44719, Addgene) in a 95:5 ratio using Lipofectamine 3000 (L3000-001, Life Technologies) according to the manufacturer's instructions. The Cas9 sgRNA vector was a gift from Su-Chun Zhang, and pCas9_GFP was a gift from Kiran Musunuru [22, 23]. Then, transfected cells were seeded into 96-well plates at a ratio of less than 1 cell per well to ensure that every well contained a single unique cell. The cells were grown for $\sim 2$ weeks and moved into 24-well plates separately. After the cells were sufficiently grown, genomic DNA (gDNA) was extracted using a Wizard Genomic DNA Purification Kit (A1125, Promega). Then, gDNA was amplified by PCR with target-specific primers and sequenced to check properly generated deletions. Confirmed cells were moved and grown in $100 \mathrm{~mm}$ dishes, and the KO of HCC gene expression was confirmed by qRT-PCR.

\section{Knockdown of eRNA using ASO}

Locked nucleic acid (LNA)-modified ASOs complementary to eRNA of MYC were designed from Antisense LNA GapmeRs (Qiagen). The ASOs were purchased from Qiagen. The sequences are listed in Additional file 1: Table 3. For the transfection of Huh7 cells, ASOs were mixed with RNAiMAX in serum-free OptiMEM (Gibco). At varying concentrations of ASOs, dissolved Opti-MEM was added, and the cells were incubated in a growth medium for $4 \mathrm{~h}$ at $37^{\circ} \mathrm{C}$ and $5 \% \mathrm{CO}_{2}$. For total RNA extraction, the cells were harvested $48 \mathrm{~h}$ posttransfection.

\section{Colony formation assay}

R3-deleted and WT Huh7 cells were seeded on 6-well plates (SPL) in growth media at a density of 2500 cells/well and incubated in a $\mathrm{CO}_{2}$ incubator for 10 days. Then, the cells were washed with PBS, fixed with $4 \%$ paraformaldehyde for 20 min and washed once with PBS. The cells were stained with $1 \%$ crystal violet (Sigma) for $30 \mathrm{~min}$. After crystal violet was removed, the plates were washed with DW for 5 min and dried. The stained cells were analyzed for colony formation rates using ImageJ (Bethesda).

\section{Sphere formation assay}

Cells were seeded on 24-well ultralow attachment culture dishes (Corning) in growth media at a density of 1000 cells/well. DMEM/F12 serum-free medium (Gibco) contained 2 mM L-glutamine, $1 \%$ sodium pyruvate (Invitrogen), $100 \mathrm{U} / \mathrm{ml}$ penicillin, and $100 \mu \mathrm{g} / \mathrm{ml}$ streptomycin supplemented with $20 \mathrm{ng} / \mathrm{ml}$ epithelial growth factor (Invitrogen), $10 \mathrm{ng} / \mathrm{ml}$ fibroblast growth factor-2 (Invitrogen), N2 (R\&D Systems), and B27 (Invitrogen). The cells were incubated in a $\mathrm{CO}_{2}$ incubator for one to two weeks, and oncosphere cells over $100 \mu \mathrm{m}$ were counted with JuLI Br (NanoEnTek).

\section{Statistical analysis}

The data are presented as the mean \pm standard deviation (SD) of the mean. All statistical analyses were performed using the IBM SPSS Statistics 26.0 program (IBM). We used one-way analysis of variance 
followed by Tukey's honestly significant difference post hoc test. $p$ values $<0.05$ were considered significant.

\section{Results}

\section{Effects of BET inhibition on HCC cell proliferation and MYC expression}

To study the effects of enhancer activity inhibition on HCC cells, we incubated them with BET inhibitors (JQ1 or OTX-015) for different durations ( $4 \mathrm{~h}, 24 \mathrm{~h}, 48 \mathrm{~h}$, and $72 \mathrm{~h}$ ). Cell proliferation significantly reduced Huh7 cells after $48 \mathrm{~h}$ and HepG2 cells after $24 \mathrm{~h}$ of BET inhibitor treatment (Fig. 1A). Moreover, we performed a 5-ethynyl-2'-deoxyuridine (EdU) proliferation assay of HCC cell lines treated with a BET inhibitor $(5 \mu \mathrm{M})$ for $24 \mathrm{~h}$. We found that BET inhibition decreased the proportion of EdU-positive cells for $24 \mathrm{~h}$, indicating that BET inhibition reduced the proliferation of HCC cell lines (Fig. 1B). JQ1 and OTX-015 are well-known small-molecule inhibitors that prevent BRD4, a member of the BET protein family, which is required to maintain SE activation [24]. BRD4 is involved in transcription by interacting with TFs and chromatin remodeling proteins under active SE [25]. HepG2 cells were treated for $24 \mathrm{~h}$ with BET inhibitors at $5 \mu \mathrm{M}$ to determine the change in $M Y C$ and vascular endothelial growth factor A (VEGFA) mRNA expression levels. As $M Y C$ mRNA expression was reduced to approximately $70 \%$, the mRNA expression of the target genes of MYC and VEGFA [26] was also reduced by JQ1 and OTX-015 treatment (Fig. 1C). Furthermore, we analyzed the gene expression pattern in response to RNA transcription inhibition, such as p300/cAMP response element-binding (CREB)-binding protein (CBP) inhibitor (C646, $50 \mu \mathrm{M}, 24 \mathrm{~h}$ ) and RNAP $\otimes$ transcription elongation inhibitor 5,6-dichloro-1- $\beta_{-}$-ribofuranosylbenzimidazole (DRB, $50 \mu \mathrm{M}, 24$ h). C646 treatment significantly reduced the expression of $M Y C$ but not that of VEGFA. DRB treatment significantly reduced the expression of MYC and VEGFA (Fig. 1D).

These results suggest that the proliferation of HCC cells and the expression of oncogenes and tumor suppressor genes were inhibited in BET inhibitor-treated cells, as expected. Furthermore, the effect of BET inhibitors on reducing $M Y C$ expression is closely related to RNA transcription inhibition.

\section{Identification of a distal MYC enhancer in HCC cells}

We examined ENCODE ChIP-seq data and GRO-seq data of the HepG2 cell line on the MYC locus (Fig. 2). We found that the downstream regions of $M Y C$ were more enriched for H3K27ac, a histone mark for an active enhancer in HCC cells. Using the GRO-seq peak (GSE92375) (Liivrand et al., 2017; Benhammou et al., 2019), H3K27ac ChIP-seq peak (GSE29611) [2], and p300 ChIP-seq (GSE32465) [27] at the UCSC Genome Browser (http://genome.ucsc.edu), six putative enhancer loci of the MYC gene were identified (R1-6) (Fig. 2A, Additional file 1: Table 4). More specifically, we set the H3K27ac enrichment level above 50 and the GRO-seq level, meaning eRNA expression, above 20 to reflect the effect on eRNA. LUAD-R3 is a superenhancer (SE) of MYC that actively regulates MYC expression in lung adenocarcinoma cells, and LUAD-R4 has low activity [6]. To seek direct functional evidence for the suspected enhancer activities in HCC cells, we examined the activity of a luciferase reporter in transiently transfected HCC cells. Each 
enhancer region was cloned into the minimal promoter vector pGL4.26 immediately upstream of the luciferase gene for this assay. As expected, transfection of R2 and R3 increased luciferase activity by approximately 10-fold. However, enhanced activity was not observed when LUAD-R3 and LUAD-R4 were transfected. Furthermore, we cloned a few constructs containing 500 bp fragments in the R2 and R3 regions and analyzed them for luciferase activity. The R2-3-containing plasmid showed the highest enhancer activity in HCC cells, and the R3-2- and R3-3-containing plasmids were most highly expressed in HCC cells. Our results suggest that R2 (R2-3) and R3 (R3-2 and R3-3) can be considered candidate regulators of the transcriptional activation of $M Y C$ in $\mathrm{HCC}$ cells.

\section{eRNA of putative MYC enhancers in HCC cells}

Next, we analyzed R2 and R3 regions of enhancer RNA (eRNA) using qRT-PCR (Additional file 1: Table 1). Using analysis of newly synthesized RNA with GRO-seq peaks, six different sets of primers were designed to analyze eRNA expression at putative enhancer regions (Fig. 3A). qRT-PCR detected a significant reduction in sense eRNA expression in the RNAP $\square$ transcription elongation inhibitor DRB in regions R2, R3, R4, and R6 but not R1 and R5 (Fig. 3B). R2 and R3 were, therefore, further studied for expression changes through treatment with BET inhibitors. As expected, eRNA expression in regions R2 and R3 was significantly decreased in HCC cells treated with BET inhibitors (Fig. 3C). Additionally, R1, R4, R5, and R6 eRNAs were decreased in BET inhibitor-treated HCC cells (Fig. S1). Together, these results indicate that there is a correlation between the activity of enhancers and eRNA transcription. From this, it can be inferred that BET inhibitors suppress MYC expression through the regulation of eRNA expression.

\section{Disruption of MYC enhancers affects MYC-related gene expression and cell growth in HCC cells}

Since our eRNA expression experiments showed enhancer activity of R3, we tested whether deletion of the R3 region regulates $M Y C$ gene expression. We established the deletion of the R3 region in the Huh7 cell line using the CRISPR-Cas9 system. The targeted sequences are located in R3 (Chr 8: 128,556,059128,557,653) of the MYC gene downstream (Additional file 1: Table 3). Huh7 cells were transfected with each target gRNA plasmid and the Cas9 plasmid and sorted into single unique cells. After sufficient growth of the selected cells, PCR using genomic DNA revealed a deletion of the R3 region. Genomic DNA sequencing revealed a 400 bp deletion on Chr 8:128,556,578 - 128,556,952 (Fig. 4A). Huh7 cells transfected with CRISPR-Cas 9 constructs showed R3 regions deletion of approximately $350 \mathrm{bp}$ in length in the R3 region (Fig. 4B). Most MYC enhancer-knockout cells died, which made experimental verification difficult. Therefore, we used $M Y C$ enhancer knockout cells that were coexistent with wild-type cells within 10 passages. R3-deleted cells had reduced MYC gene expression relative to wild-type cells (Fig. 4C). Using qRT-PCR analysis, we revealed that R2 eRNA and R3 eRNA expression was significantly decreased in the R3-deleted cells (Fig. 4D). To assess the effect of R3 deletion on IncRNA expression of MYC located downstream within Chr 8: 127,735,502 - 130,060,473, the expression levels of PVT1, CCAT1, and FAM49B were analyzed by qRT-PCR. LncRNA CCAT1 (Located in Chr 8: 127,207,382-127,219,268), and FAM49B (located in Chr 8: 129,841,470 - 130,016,672) were reduced in R3-deleted cells, whereas the expression level of PVT1 (located on Chr 8: 127,795,799-128,101,256) were almost identical in R3-deleted cells and 
the wild-type cells (Fig. 4E). We further analyzed the expression of genes regulated by MYC, such as IRF2 and TERT. We found that the expression of IRF2 was upregulated in R3-deleted cells, whereas the expression of TERT was downregulated (Fig. 4F). Furthermore, we performed a cell proliferation assay and colony formation assay of R3-deleted Huh7 cells. We found that the R3-deleted cells had decreased proliferation and colony formation ability (Fig. 4G, H). The results confirm that enhancer deletion influences cancer cell growth by reducing MYC expression [28]. Deletion in the R3 region reduced the sphere formation of Huh7 cells (Fig. 4I).

\section{Inhibition of MYC eRNA represents an effect equivalent to MYC enhancer disruption}

To further confirm the functional role of $M Y C$ eRNA, antisense oligonucleotides (ASOs) were designed to bind an eRNA at R2 and R3 (MYC-R2 and MYC-R3, respectively) (Additional file: Table 1). To validate the predicted eRNA reduction, we delivered $125 \mathrm{pmol}$ ASO to HCC cells by transfection and analyzed the expression levels of eRNAs and MYC by qRT-PCR. The eRNA expression of R2 was specifically decreased in HCC-transfected ASO-R2 cells (Fig. 5B). The eRNA expression of MYC-R3 was specifically decreased in HCC cells transfected with ASO-R3 (Fig. 5C). Both ASO-R2- and ASO-R3-transfected cells had significantly reduced $M Y C$ gene expression relative to the ASO-NC-transfected cells (Fig. 5D). As a result of confirming the eRNA inhibitory effect, when $M Y C$-R2 was inhibited, the expression of IncRNA was confirmed as in MYC-R3 deletion, as shown in Fig. 4, and PVT1 expression increased when MYC-R3 was inhibited (Fig. 5E). In addition, the expression of ICAM1 and IRF2, which MYC regulates, was decreased (Fig. 5F) [29]. We further analyzed the proliferation and sphere-forming ability of HCC cells after treatment with ASO. As eRNA expression of $M Y C-\mathrm{R} 2$ and R3 decreased, it significantly reduced cell proliferation compared to NC ASO-treated HCC cells (Fig. 5G). In addition, colony formation assays showed that ASOR3 treatment negatively affected cell adhesion and growth initiation (Fig. S2). The sphere-forming ability was also decreased in ASO-treated HCC cells, especially in ASO-R2 cells (Fig. 5H). The results shown in Fig. 5 show that inhibition of eRNA reduces MYC expression without direct deletion of DNA and suppresses cell proliferation and stemness of HCC cells. Additionally, MYC is involved in regulating the self-renewal and survival of glioma cancer stem cells (CSCs) and colon CSCs as a key regulator of stem cell biology [30-32]. Our results indicate that MYC regulation affects the stemness of HCC cells.

\section{Discussion}

$\mathrm{HCC}$ is the most common type of primary liver cancer. HCC is prevalent cancer globally and a leading cause of cancer-related death [33,34]. Significant genetic and epigenetic alterations exist in HCC. Its accumulation in key genes involved in cell survival, proliferation, apoptosis, and metastasis leads to carcinogenesis [35]. The dysregulation of MYC plays a vital role in proliferation and invasion, including tumor initiation and progression in HCC [36, 37].

The antitumor effect of BET inhibitors such as JQ1 has been proven in various cancer cells, including HCC cells [38-40]. The antitumor effect is due to the reduction of oncogenes, including MYC. Several studies have already confirmed the inhibitory effect of tumor growth by inhibiting $M Y C$ expression 
through BRD4 inhibitors [19, 39, 41, 42]. Despite the effects of BET inhibitors on MYC downregulation, they are not appropriate or effective for use in patients due to various side effects, such as toxicity and resistance.

Recent studies have shown that RNA transcripts from SEs, large clusters of enhancers, are directly or indirectly involved in oncogene expression, cell growth, metastasis, drug resistance, etc. [43]. The enhancer is a DNA region in which the E1A-binding proteins p300 (p300), RNAP区, and TFs are enriched through increased accessibility caused by histone modification. SEs help the transcriptional activation of the promoter directly through looping or indirectly affect transcription by expressing the eRNA $[3,44]$. Previous studies have revealed that the presence of SEs, including cancer-specific enhancers and the expression of eRNA in cancer cells; accordingly, the roles of SEs in the abnormal expression of oncogenes have been elucidated $[5,45]$. In addition, since the binding of lineage-specific TFs affects the tumorigenesis function of the enhancer, it can be seen that the enhancer activity is also cancer typespecific [46]. Therefore, we used ChIP-seq data with HCC cells and used global run-on sequencing (GROseq) data that included nascent transcripts to identify eRNA expression, an active enhancer marker [4, 47]. Considering these data, we found that specific MYC enhancers R2 and R3 in HCC cells are significantly different from those in other cancers in terms of their location and activity $[5,6,48]$. In acute myeloid leukemia (AML), MYCSE, consisting of five distinct small enhancers located 1.7 Mb downstream of the MYC promoter regulates MYC expression [45]. In colorectal cancer (CRC) and prostate cancer, the MYC enhancer located $335 \mathrm{~kb}$ upstream of MYC regulates MYC expression by interacting with its promoter [19]. In addition, the Zhang group deleted approximately $1.5 \mathrm{~kb}$ of the MYC enhancer located $450 \mathrm{~kb}$ toward the $3^{\prime}$ end in lung adenocarcinoma cells. MYC expression was reduced by $70 \%$, and clonogenic growth was inhibited by approximately $50 \%$. In HCC, we confirmed the reduction of MYC expression and inhibition of cell growth by more than one-half upon deletion of a $400 \mathrm{bp} \mathrm{HCC}$-specific MYC enhancer located $800 \mathrm{~kb}$ downstream of MYC.

eRNAs are generally upregulated in various cancers compared to normal tissues, and they can be used as pan-cancer diagnostic markers [49]. In addition, tissue-specific highly expressed eRNAs, such as CCAT1 in colorectal cancer and androgen receptor (AR)-induced Kallikrein-related peptidase 3 (KLK3) eRNA (KLK3e) in prostate cancer, are considered new targets for treating various cancers $[9,50,51]$. Although the function of eRNAs has not been fully elucidated, eRNA depletion reduces the transcription of target genes by affecting alterations in chromatin structure and contributing to transcriptional initiation of target genes [52]. eRNA transcription can be regulated by inhibiting enhancer activity or effectively targeting ASO to control target gene expression and cancer cell progression $[13,53]$.

Inhibition of MYC-IEANC RNAs using ASO effectively perturbs enhancers to inhibit HCC cell progression, suggesting that eRNAs can be helpful as therapeutic targets. Several studies have reported the therapeutic effect of eRNA depletion on cancer [53-55]. In addition, Epstein-Barr virus (EBV) superenhancer (ESE) RNAs facilitated the expression of the MYC oncogene in lymphoma and showed a therapeutic effect on EBV-related malignancies by targeting ESE eRNA [55]. Similarly, in our results, targeting MYC-IEANC RNAs transcribed from MYC enhancers R2 and R3 in HCC cells confirmed an 
effective decrease in MYC expression along with proliferation and stemness reduction in HCC cells, suggesting a therapeutic effect on HCC. The study that downregulation of MYC, a marker of stemness, suppressed spheroid growth of colon CSCs and tumor growth in vivo can support our suggestion [32].

\section{Conclusion}

In this study, we identified the putative MYC enhancer in HCC cells. Enhancer activity and eRNA transcription were analyzed to determine the region involved in MYC expression, and it was confirmed that inhibiting enhancers and eRNAs suppressed the proliferation and reduced sphere formation of HCC cells. These results implied that the inhibition of eRNA by ASO treatment had corresponding effects on the deletion of $M Y C$ enhancers. Thus, our study suggests that for HCC, a strategy for reducing MYC expression through specific targeting with ASO has therapeutic potential without the side effects of gene editing.

\section{List Of Abbreviations}

The abbreviations used are; HCC, Hepatocellular carcinoma; eRNA, enhancer RNA; ASO, Antisense oligonucleotide; SE, superenhancer; GRO-seq, global run-on sequencing; H2K27ac, acetylated H3 lysine 27; Chr, Chromosome; CSC, cancer stem cell; qRT-PCR, quantitative reverse transcription-polymerase chain reaction

\section{Declarations}

\section{Ethics approval and consent to participate}

Not applicable

\section{Consent for publication}

Not applicable

\section{Availability of data and materials}

Not applicable

\section{Competing interests}

The authors declare that they have no conflicts of interest with the contents of this article.

\section{Funding}

This work was supported by the National Research Foundation of Korea (NRF) Grants 2017M3A9G7073033, and 2020R1A2C1014193 (to Y. G. C.), 2016R1D1A1B04934970 (to K. H. J.), and 2014M3C9A3064693 (to Y. S. L.) from the Korean government. 


\section{Authors' Contributions}

H. I. C. designed and performed experiments, analyzed and interpreted the data, and prepared the manuscript; J. C. C. and Y. S. L. analyzed next-generation sequencing and bioinformatics data; K. H. J. designed experiments, financial support, analyzed and interpreted the data, prepared and edited the manuscript; Y. G. C. designed experiments, financial support, analyzed and interpreted next-generation sequencing and bioinformatics data, final approval of the manuscript, edited the manuscript.

\section{Acknowledgements}

Not applicable

\section{Author information}

\section{Affiliations}

Department of Bionanotechnology, Hanyang University, Seoul, 04673, Republic of Korea

Hae In Choi, Young Gyu Chai

Department of Molecular \& Life Science, Hanyang University, Ansan, 15588, Republic of Korea

Young Gyu Chai

College of Veterinary Medicine, Seoul National University, Seoul, 08826, Republic of Korea Jin Choul Chai, Young Seek Lee

Convergence Technology Campus of Korea Polytechnic II, Incheon, 21417, Republic of Korea Kyoung Hwa Jung

\section{References}

1. Jaenisch R, Bird A. Epigenetic regulation of gene expression: how the genome integrates intrinsic and environmental signals. Nat Genet. 2003;33 Suppl:245-54.

2. Consortium EP. An integrated encyclopedia of DNA elements in the human genome. Nature. 2012;489:57-74.

3. Sur I, Taipale J. The role of enhancers in cancer. Nat Rev Cancer. 2016;16:483-93.

4. Andersson R, Gebhard C, Miguel-Escalada I, Hoof I, Bornholdt J, Boyd M, Chen Y, Zhao X, Schmidl C, Suzuki T, et al. An atlas of active enhancers across human cell types and tissues. Nature. 2014;507:455-61.

5. Loven J, Hoke HA, Lin CY, Lau A, Orlando DA, Vakoc CR, Bradner JE, Lee TI, Young RA. Selective inhibition of tumor oncogenes by disruption of super-enhancers. Cell. 2013;153:320-34. 
6. Zhang X, Choi PS, Francis JM, Imielinski M, Watanabe H, Cherniack AD, Meyerson M. Identification of focally amplified lineage-specific super-enhancers in human epithelial cancers. Nat Genet. 2016;48:176-82.

7. Hamdan FH, Johnsen SA. Perturbing Enhancer Activity in Cancer Therapy. Cancers (Basel). 2019; 11.

8. Heinz S, Romanoski CE, Benner C, Glass CK. The selection and function of cell type-specific enhancers. Nat Rev Mol Cell Biol. 2015;16:144-54.

9. Hsieh CL, Fei T, Chen Y, Li T, Gao Y, Wang X, Sun T, Sweeney CJ, Lee GS, Chen S, et al. Enhancer RNAs participate in androgen receptor-driven looping that selectively enhances gene activation. Proc Natl Acad Sci U S A. 2014;111:7319-24.

10. Arnold PR, Wells AD, Li XC. Diversity and Emerging Roles of Enhancer RNA in Regulation of Gene Expression and Cell Fate. Front Cell Dev Biol. 2019;7:377.

11. Lee JH, Xiong F, Li W. Enhancer RNAs in cancer: regulation, mechanisms and therapeutic potential. RNA Biol. 2020;17:1550-9.

12. Lam MT, Cho H, Lesch HP, Gosselin D, Heinz S, Tanaka-Oishi Y, Benner C, Kaikkonen MU, Kim AS, Kosaka $\mathrm{M}$, et al. Rev-Erbs repress macrophage gene expression by inhibiting enhancer-directed transcription. Nature. 2013;498:511-5.

13. Pan CW, Wen S, Chen L, Wei Y, Niu Y, Zhao Y. Functional roles of antisense enhancer RNA for promoting prostate cancer progression. Theranostics. 2021;11:1780-94.

14. Scoles DR, Minikel EV, Pulst SM. Antisense oligonucleotides: A primer. Neurol Genet. 2019;5:e323.

15. Pelengaris S, Khan M, Evan G. c-MYC: more than just a matter of life and death. Nat Rev Cancer. 2002;2:764-76.

16. Adhikary S, Eilers M. Transcriptional regulation and transformation by Myc proteins. Nat Rev Mol Cell Biol. 2005;6:635-45.

17. Gabay M, Li Y, Felsher DW. MYC activation is a hallmark of cancer initiation and maintenance. Cold Spring Harb Perspect Med. 2014; 4.

18. Lancho O, Herranz D. The MYC Enhancer-ome: Long-Range Transcriptional Regulation of MYC in Cancer. Trends Cancer. 2018;4:810-22.

19. Ahmadiyeh N, Pomerantz MM, Grisanzio C, Herman P, Jia L, Almendro V, He HH, Brown M, Liu XS, Davis $\mathrm{M}$, et al. 8q24 prostate, breast, and colon cancer risk loci show tissue-specific long-range interaction with MYC. Proc Natl Acad Sci U S A. 2010;107:9742-6.

20. Hnisz D, Abraham BJ, Lee TI, Lau A, Saint-Andre V, Sigova AA, Hoke HA, Young RA. Super-enhancers in the control of cell identity and disease. Cell. 2013;155:934-47.

21. Oktay Y, Ulgen E, Can O, Akyerli CB, Yuksel S, Erdemgil Y, Durasi IM, Henegariu OI, Nanni EP, Selevsek $\mathrm{N}$, et al. IDH-mutant glioma specific association of rs55705857 located at 8q24.21 involves MYC deregulation. Sci Rep. 2016;6:27569.

22. Chen $Y$, Cao J, Xiong M, Petersen AJ, Dong Y, Tao Y, Huang CT, Du Z, Zhang SC. Engineering Human Stem Cell Lines with Inducible Gene Knockout using CRISPR/Cas9. Cell Stem Cell. 2015;17:233-44. 
23. Ding Q, Regan SN, Xia Y, Oostrom LA, Cowan CA, Musunuru K. Enhanced efficiency of human pluripotent stem cell genome editing through replacing TALENs with CRISPRs. Cell Stem Cell. 2013;12:393-4.

24. Bhagwat AS, Roe JS, Mok BYL, Hohmann AF, Shi J, Vakoc CR. BET Bromodomain Inhibition Releases the Mediator Complex from Select cis-Regulatory Elements. Cell Rep. 2016;15:519-30.

25. Donati B, Lorenzini E, Ciarrocchi A. BRD4 and Cancer: going beyond transcriptional regulation. Mol Cancer. 2018;17:164.

26. Knies-Bamforth UE, Fox SB, Poulsom R, Evan GI. \& Harris AL. c-Myc interacts with hypoxia to induce angiogenesis in vivo by a vascular endothelial growth factor-dependent mechanism. Cancer research. 2004;18:6563-70.

27. Gertz J, Savic D, Varley KE, Partridge EC, Safi A, Jain P, Cooper GM, Reddy TE, Crawford GE, Myers RM. Distinct properties of cell-type-specific and shared transcription factor binding sites. Mol Cell. 2013;52:25-36.

28. Tak YG, Hung Y, Yao L, Grimmer MR, Do A, Bhakta MS, O'Geen H, Segal DJ, Farnham PJ. Effects on the transcriptome upon deletion of a distal element cannot be predicted by the size of the H3K27Ac peak in human cells. Nucleic Acids Res. 2016;44:4123-33.

29. Fernandez PC, Frank SR, Wang L, Schroeder M, Liu S, Greene J, Cocito A, Amati B. Genomic targets of the human c-Myc protein. Genes Dev. 2003;17:1115-29.

30. Takahashi K, Yamanaka S. Induction of pluripotent stem cells from mouse embryonic and adult fibroblast cultures by defined factors. Cell. 2006;126:663-76.

31. Wang J, Wang H, Li Z, Wu Q, Lathia JD, McLendon RE, Hjelmeland AB, Rich JN. c-Myc is required for maintenance of glioma cancer stem cells. PLoS One. 2008;3:e3769.

32. Zhang HL, Wang P, Lu MZ, Zhang SD, Zheng L. c-Myc maintains the self-renewal and chemoresistance properties of colon cancer stem cells. Oncol Lett. 2019;17:4487-93.

33. Balogh J, Victor D 3rd, Asham EH, Burroughs SG, Boktour M, Saharia A, Li X, Ghobrial RM, Monsour HP. Jr.. Hepatocellular carcinoma: a review. J Hepatocell Carcinoma. 2016;3:41-53.

34. Yang Y, Liu L, Li M, Cheng X, Fang M, Zeng Q, Xu Y. The chromatin remodeling protein BRG1 links ELOVL3 trans-activation to prostate cancer metastasis. Biochim Biophys Acta Gene Regul Mech. 2019;1862:834-45.

35. Liu M, Jiang L, Guan XY. The genetic and epigenetic alterations in human hepatocellular carcinoma: a recent update. Protein Cell. 2014;5:673-91.

36. Zender L, Villanueva A, Tovar V, Sia D, Chiang DY, Llovet JM. Cancer gene discovery in hepatocellular carcinoma. Journal of hepatology. 2010;6:921-9.

37. Zheng K, Cubero FJ, Nevzorova YA. c-MYC-Making Liver Sick: Role of c-MYC in Hepatic Cell Function, Homeostasis and Disease. Genes (Basel). 2017; 8.

38. Bechter O, Schoffski P. Make your best BET: The emerging role of BET inhibitor treatment in malignant tumors. Pharmacol Ther. 2020;208:107479. 
39. Li GQ, Guo WZ, Zhang Y, Seng JJ, Zhang HP, Ma XX, Zhang G, Li J, Yan B, Tang HW, et al. Suppression of BRD4 inhibits human hepatocellular carcinoma by repressing MYC and enhancing BIM expression. Oncotarget. 2016;7:2462-74.

40. Spriano F, Stathis A, Bertoni F. Targeting BET bromodomain proteins in cancer: The example of lymphomas. Pharmacol Ther. 2020;215:107631.

41. Delmore JE, Issa GC, Lemieux ME, Rahl PB, Shi J, Jacobs HM, Kastritis E, Gilpatrick T, Paranal RM, Qi J, et al. BET bromodomain inhibition as a therapeutic strategy to target c-Myc. Cell. 2011;146:90417.

42. Sengupta D, Kannan A, Kern M, Moreno MA, Vural E, Stack B Jr, Suen JY, Tackett AJ, Gao L. Disruption of BRD4 at H3K27Ac-enriched enhancer region correlates with decreased c-Myc expression in Merkel cell carcinoma. Epigenetics. 2015;10:460-6.

43. Wang Y, Nie H, He X, Liao Z, Zhou Y, Zhou J, Ou C. The emerging role of super enhancer-derived noncoding RNAs in human cancer. Theranostics. 2020;10:11049-62.

44. Kim TK, Hemberg M, Gray JM, Costa AM, Bear DM, Wu J, Harmin DA, Laptewicz M, Barbara-Haley K, Kuersten $\mathrm{S}$, et al. Widespread transcription at neuronal activity-regulated enhancers. Nature. 2010;465:182-7.

45. Shi J, Vakoc CR. The mechanisms behind the therapeutic activity of BET bromodomain inhibition. Mol Cell. 2014;54:728-36.

46. Pomerantz MM, Li F, Takeda DY, Lenci R, Chonkar A, Chabot M, Cejas P, Vazquez F, Cook J, Shivdasani RA, et al. The androgen receptor cistrome is extensively reprogrammed in human prostate tumorigenesis. Nat Genet. 2015;47:1346-51.

47. Park A, Oh S, Jung KL, Choi UY, Lee HR, Rosenfeld MG, Jung JU. Global epigenomic analysis of KSHV-infected primary effusion lymphoma identifies functional MYC superenhancers and enhancer RNAs. Proc Natl Acad Sci U S A. 2020;117:21618-27.

48. Schuijers J, Manteiga JC, Weintraub AS, Day DS, Zamudio AV, Hnisz D, Lee TI, Young RA. Transcriptional Dysregulation of MYC Reveals Common Enhancer-Docking Mechanism. Cell Rep. 2018;23:349-60.

49. Kaczkowski B, Tanaka Y, Kawaji H, Sandelin A, Andersson R, Itoh M, Lassmann T, Hayashizaki Y, Carninci P, Forrest AR, Consortium F. Transcriptome Analysis of Recurrently Deregulated Genes across Multiple Cancers Identifies New Pan-Cancer Biomarkers. Cancer Res. 2016;76:216-26.

50. McCleland ML, Mesh K, Lorenzana E, Chopra VS, Segal E, Watanabe C, Haley B, Mayba O, Yaylaoglu M, Gnad F, Firestein R. CCAT1 is an enhancer-templated RNA that predicts BET sensitivity in colorectal cancer. J Clin Invest. 2016;126:639-52.

51. Oh S, Shao J, Mitra J, Xiong F, D'Antonio M, Wang R, Garcia-Bassets I, Ma Q, Zhu X, Lee JH, et al. Enhancer release and retargeting activates disease-susceptibility genes. Nature. 2021.

52. Pnueli L, Rudnizky S, Yosefzon Y, Melamed P. RNA transcribed from a distal enhancer is required for activating the chromatin at the promoter of the gonadotropin alpha-subunit gene. Proc Natl Acad Sci U S A. 2015;112:4369-74. 
53. Zhang Z, Lee JH, Ruan H, Ye Y, Krakowiak J, Hu Q, Xiang Y, Gong J, Zhou B, Wang L, et al. Transcriptional landscape and clinical utility of enhancer RNAs for eRNA-targeted therapy in cancer. Nat Commun. 2019;10:4562.

54. Jiao W, Chen Y, Song H, Li D, Mei H, Yang F, Fang E, Wang X, Huang K, Zheng L, Tong Q. HPSE enhancer RNA promotes cancer progression through driving chromatin looping and regulating hnRNPU/p300/EGR1/HPSE axis. Oncogene. 2018;37:2728-45.

55. Liang J, Zhou H, Gerdt C, Tan M, Colson T, Kaye KM, Kieff E, Zhao B. Epstein-Barr virus superenhancer eRNAs are essential for MYC oncogene expression and lymphoblast proliferation. Proc Natl Acad Sci U S A. 2016;113:14121-6.

\section{Tables}

Table 1. Primer sequences used for qRT-PCR

\begin{tabular}{|c|c|c|}
\hline Gene & Forward $\left(5^{\prime}-3^{\prime}\right)$ & Reverse $\left(5^{\prime}-3^{\prime}\right)$ \\
\hline GAPDH & AAGGTCGGAGTCAACGGATT & СTCCTGGAAGATGGTGATGG \\
\hline MYC & GGCTCCTGGCAAAAGGTCA & AGTTGTGCTGATGTGTGGAGA \\
\hline DUSP9 & CAGCCGTTCTGTCACCGTC & CAAGCTGCGCTCAAAGTCC \\
\hline VEGFA & TTATGCGGATCAAACCTCACC & GAAGCTCATCTCTCCTATGTGC \\
\hline PVT1 & GCCССTTCTATGGGAATCACTA & GGGGCAGAGATGAAATCGTAAT \\
\hline CCAT1 & GGGCTGGTACAGACTAGGGA & TAAGCAGGTCAGAAAGGGCG \\
\hline FAM49B & CTCAAGATGACAAATCCTGC & CCGGTACATTGTTAATCCTC \\
\hline ICAM1 & ATGCCCAGACATCTGTGTCC & GGGGTCTCTATGCCCAACAA \\
\hline IRF2 & AATGCTGCCCCTATCAGAACG & CAGGACCGCATACTCAGGAGA \\
\hline TERT & AAATGCGGCCCCTGTTTCT & CAGTGCGTCTTGAGGAGCA \\
\hline eRNA & Forward $\left(5^{\prime}-3^{\prime}\right)$ & Reverse $\left(5^{\prime}-3^{\prime}\right)$ \\
\hline R1 & GGCTGGAGCCAGAAAGCTAA & GTCTGTGGCTCAGATGCTGT \\
\hline R2 & ACTGCAGCCACACTTCTGTT & TCTTGCTGCAATCCAGAGGG \\
\hline R3 & GCACTTGTTCCTACCTCCGC & ATGTAGTGGGACCATCACCC \\
\hline R4 & ATGCCATTTTGGGCCTTCAATAAG & TGCTACAAGTATGTGCTGACC \\
\hline R5 & GCCAGGGATGAAAAGGCCAA & TCACAGTGTAGGGGGTGTGT \\
\hline R6 & AGGTGCATTTAGCCCCAGTC & GTCTCTGGGTTCCAAGCTCC \\
\hline
\end{tabular}


Table 2. Primers for cloning

Page 16/ 25 


\begin{tabular}{|c|c|}
\hline Enhancer & Sequence $\left(5^{\prime}-3^{\prime}\right)$ \\
\hline LUAD-R3-F_Nhe囚 & ATAGCTAGCTGCCAATGGTCAGTTCTCTG \\
\hline LUAD-R3-R_Xhoه & ATACTCGAGGTCACCATTGGTACCCCAAG \\
\hline LUAD-R4-F_Sac $\rrbracket$ & ATAGAGCTCTAGCCTACAGGGACCAATGC \\
\hline LUAD-R4-R_Xho囚 & ATACTCGAGCAGCAGTGGACAAAACCAAA \\
\hline R1-F_Nhel & ATAGCTAGCGCGTTTCCTGAAACACCACC \\
\hline R1-R_Xhol & ATACTCGAGAGAGGAAGGCCATGCCTAGA \\
\hline R2-F_Nhel & ATAGCTAGCAGGTTAATGCAGAGCCTCCTC \\
\hline R2-R_Xhol & ATACTCGAGTGAAAAGTCAAGTGCCGTCTG \\
\hline R3-F_Nhel & ATAGCTAGCCCCTTTCAAGGGCAAAGACGA \\
\hline R3-R_Xhol & ATACTCGAGTGTCCTGAGAGTGGAGGCTTA \\
\hline R4-F_Nhel & ATAGCTAGCCGTCTCAGTTTCCAGAGGGTTC \\
\hline R4-R_Xhol & ATACTCGAGTCTTAGATTGAGACAGGGAGTTGA \\
\hline R5-F_Nhel & ATAGCTAGCTAGGTTGCCTGCCCTAGTAGT \\
\hline R5-R_Xhol & ATACTCGAGATTCTACCCGGTGTACAGCAC \\
\hline R6-F_Nhel & ATAGCTAGCCTTTCTGCCAGGCAACATGC \\
\hline R6-R_Xhol & ATACTCGAGTGACCACTCCATGCCAGTTC \\
\hline R2-1-F_Nhel & ATAGCTAGCAGGTTAATGCAGAGCCTCCTC \\
\hline R2-1_R_Xhol & ATACTCGAGTTAGATGAGGAAACCGAGGCAT \\
\hline R2-2-F_Nhel & ATAGCTAGCATCTGATTTCTATGAAGCTTTGAC \\
\hline R2-2-R_Xhol & ATACTCGAGCAATTTCCGAACGCTTGAGA \\
\hline R2-3-F_Nhel & ATAGCTAGCTCCTTTCTCAAGCGTTCGGA \\
\hline R2-3-R_Xhol & ATACTCGAGTGTCGGCAAGCTCAAAATGC \\
\hline R2-4-F_Nhel & ATAGCTAGCTTTAGAGCTCAAGTGCGCCA \\
\hline R2-4-R_Xhol & ATACTCGAGTGTGGCTCTTGCCTTCAGAG \\
\hline R2-5-F_Nhel & ATAGCTAGCCTTGCAGGCTGCTCTCTTTC \\
\hline R2-5-R_Xhol & ATACTCGAGTGAAAAGTCAAGTGCCGTCTG \\
\hline R3-1-F_Nhel & ATAGCTAGCCCCTTTCAAGGGCAAAGACGA \\
\hline R3-1-R_Xhol & ATACTCGAGCTGAACCCAGAAATATGAAGGAC \\
\hline
\end{tabular}




\begin{tabular}{|ll|} 
R3-2-F_Nhel & ATAGCTAGCTGGGTTTCTAGGTTTTAGTCCTTC \\
R3-2-R_Xhol & ATACTCGAGCGTGTCATTACAGTGCAGAGG \\
R3-3-F_Nhel & ATAGCTAGCCCTCTGCACTGTAATGACACGA \\
R3-3-R_Xhol & ATACTCGAGGCCTGGCAAACTTGTTTTGAGAA \\
R3-4-F_Nhel & ATAGCTAGCTAGGCACTTTCAGCTGGTCA \\
R3-4-R_Xhol & ATACTCGAGTGTCCTGAGAGTGGAGGCTTA \\
\hline
\end{tabular}

Table 3. ASO and sgRNA target sequence.

\begin{tabular}{ll} 
ASO & Sequence $\left(\mathbf{5}^{\prime} \mathbf{3}^{\prime}\right.$ ) \\
\hline ASO-R2 & CCTTCAAAGCATCACG \\
\hline ASO-R3 & CTAGTTTCCACTGACA \\
\hline sgRNA & Sequence $\left(\mathbf{5}^{\prime} \mathbf{3}^{\prime}\right.$ ) \\
\hline MYC-R3-gRNA-1 & AACTGAACCCAGAAATATGA \\
\hline MYC-R3-gRNA-2 & CTCGTGTCATTACAGTGCAG
\end{tabular}

Table 4. MYC enhancer regions

\begin{tabular}{ll} 
Enhancer & h38_DNA range \\
\hline LUAD-R3 & chr8:128176171-128177714 \\
\hline LUAD-R4 & chr8:128183039-128183988 \\
\hline R1 & chr8:128184800-128186225 \\
\hline R2 & chr8:128554145-128555945 \\
\hline R3 & chr8:128556059-128557653 \\
\hline R4 & chr8:128582625-128583855 \\
\hline R5 & chr8:128584574-128585781 \\
\hline R6 & chr8:128601617-128603273
\end{tabular}

Figures 

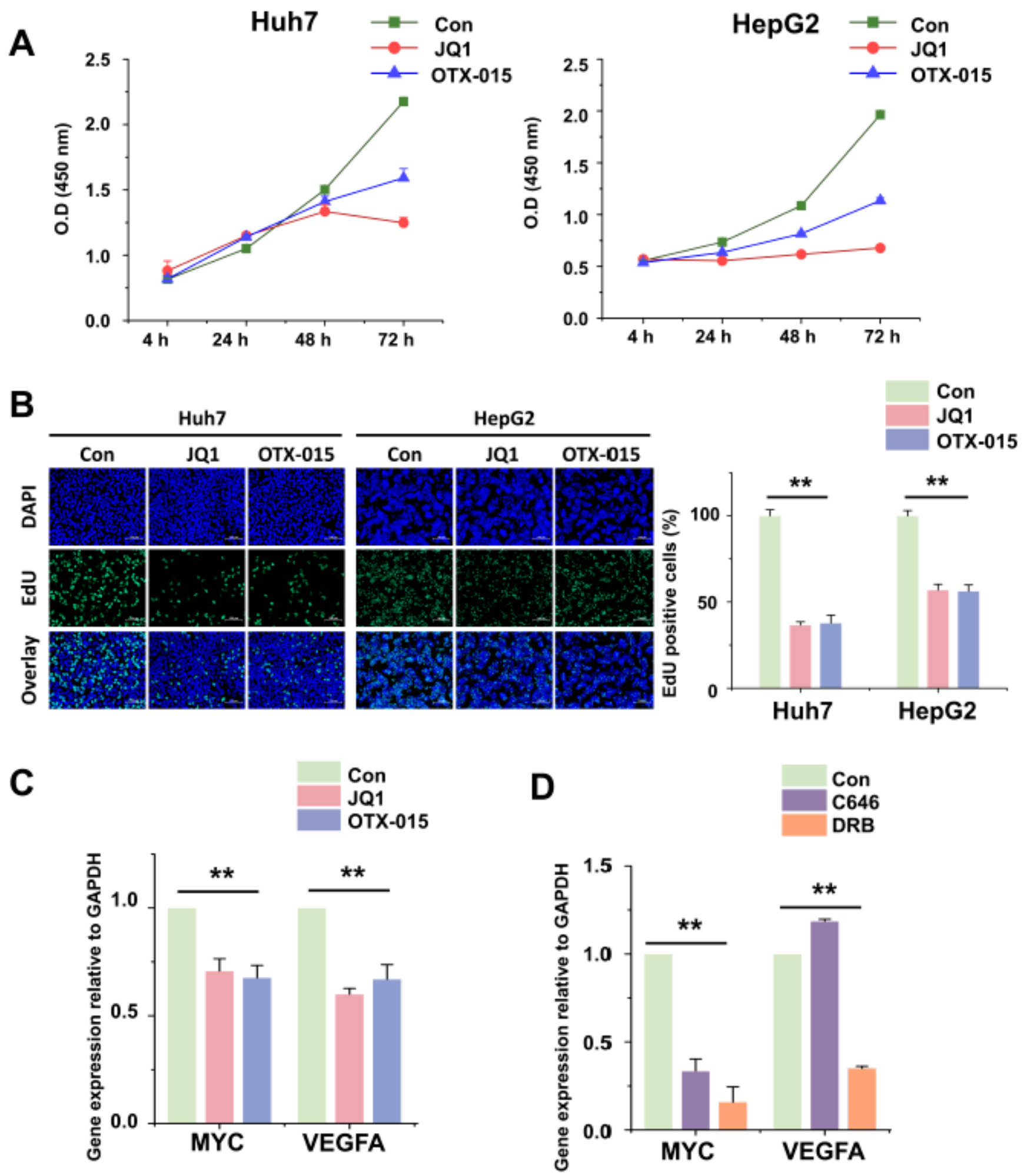

Figure 1

BET inhibitor suppresses cell viability, cell proliferation, MYC expression, and MYC target gene expression in HCC cells (A) Cell viability was determined by the WST1 assay and represented by the relative absorbance at $450 \mathrm{~nm}$. HCC cells were treated with $5 \mu \mathrm{M}$ BET inhibitors (JQ1 or OTX-015) for different durations ( $4 \mathrm{~h}, 24 \mathrm{~h}, 48 \mathrm{~h}$, and $72 \mathrm{~h}$ ). (B) The proliferation of HCC cells treated with $5 \mu \mathrm{M}$ BET inhibitors for $24 \mathrm{~h}$ was assessed by EdU assay. Representative images and the number of EdU-positive cells (\%) are 
shown. Original magnification, 400x. The data represent three biologically independent experiments. **, $p$ $<0.01$. (C) qRT-PCR analysis of MYC mRNA and MYC target genes and VEGFA mRNA in BET inhibitortreated HepG2 cells $(5 \mu \mathrm{M}, 24 \mathrm{~h})$. (D) qRT-PCR analysis of MYC mRNA and MYC target genes, VEGFA

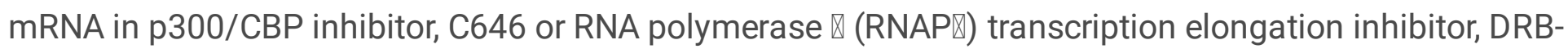
treated HepG2 cells $(50 \mu \mathrm{M}, 6 \mathrm{~h})$. The values are presented as the mean \pm SD from triplicate well measurements. *, $p<0.05$ and **, $p<0.01$.

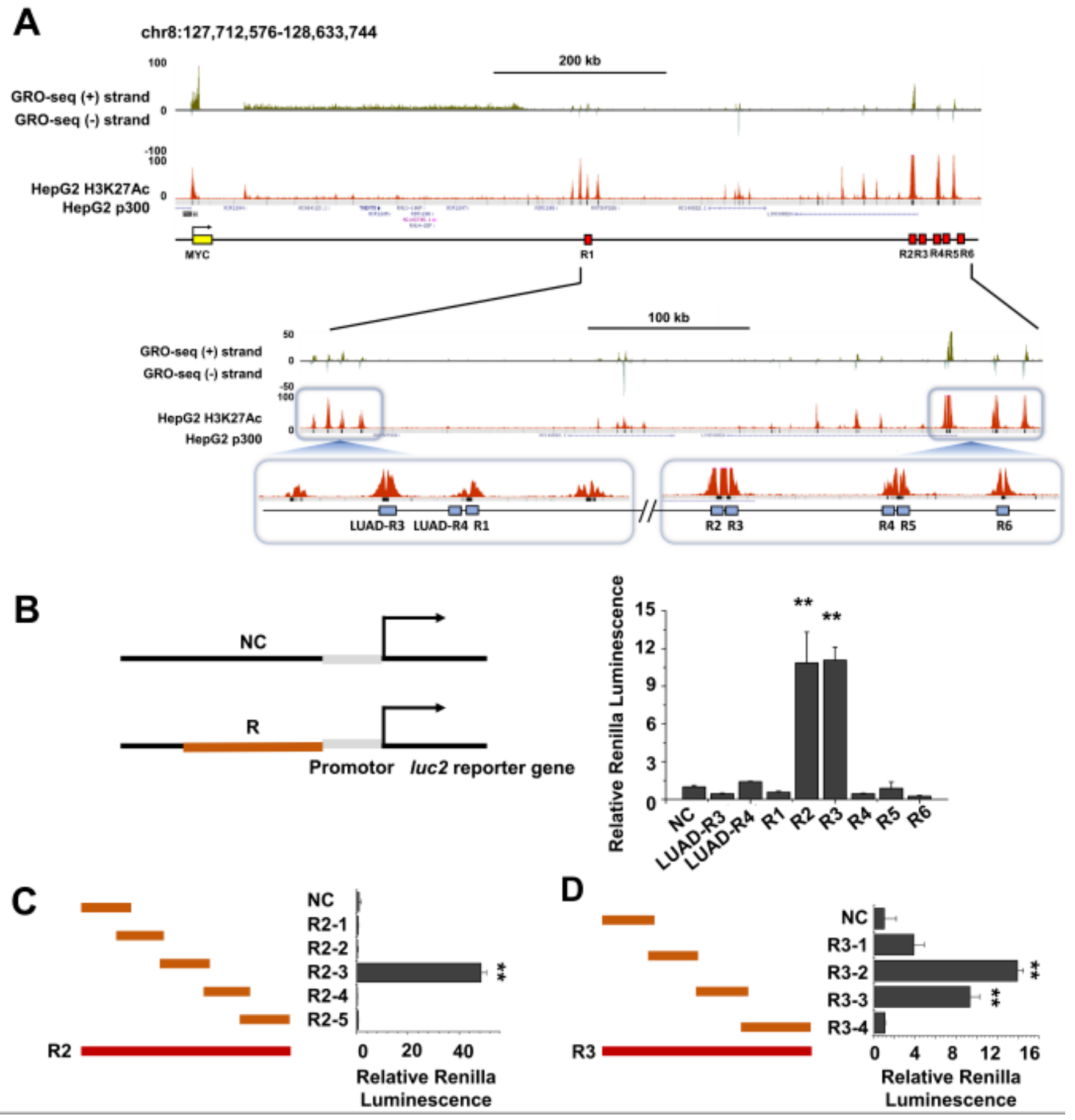

Figure 2

Identification of MYC enhancer in HCC cells (A) USCS genome browser view of the GRO-seq peak, H3K27ac enrichment, and p300 binding sites along the MYC locus. Potential MYC enhancer is indicated 
$+500 \mathrm{~kb}$ downstream (red boxes). An enlarged display of H3K27ac-enriched reads aligned to the putative enhancer regions (GRCh38/Chr 8: 127,712,576-128,633,744). Six putative enhancer regions (R1, R2, R3, $\mathrm{R} 4, \mathrm{R} 5$, and R6) located downstream of the MYC gene are numbered. (B) Luciferase assay was used to identify the regions of active enhancers for MYC expression. These six putative enhancer regions were cloned upstream of the firefly luciferase reporter (Luc2 gene). (C) The fragments of the R2 region were placed upstream of a luciferase reporter. (D) Fragments of the R3 region were placed upstream of a luciferase reporter. For each transfection, the firefly luciferase activity was normalized to that of the Renilla reniformis luciferase activity. The data are normalized to the pGL4.26 construct. The data represent three independent experiments. **, $p<0.01$.
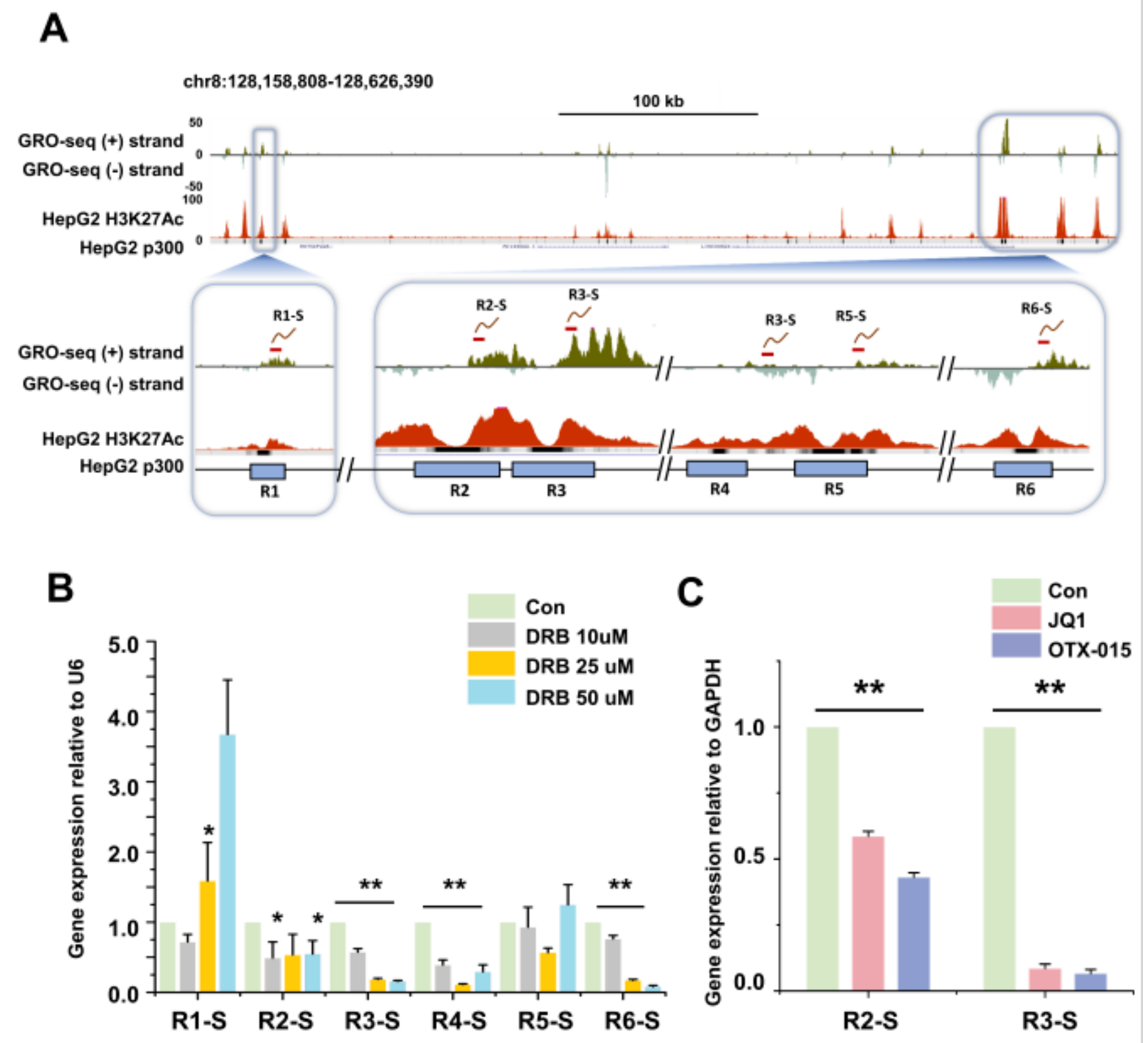

\section{Figure 3}

eRNA expression of MYC enhancer regions in HCC cells (A) USCS genome browser view of the GRO-seq peak, H3K27ac enrichment, and p300 binding sites along the MYC locus. An enlarged display of GRO-seq 
peak (+ strand and - strand) and H3K27ac enriched reads aligned to the putative enhancer regions (GRCh38/Chr 8: 128,158,808-128,626,390). eRNA primer design in six putative enhancer regions (R1, R2, R3, R4, R5, and R6). The red line above the GRO-seq (+) strand indicates target eRNA. (B) qRT-PCR of eRNA transcription levels in HepG2 cells treated with different concentrations of DRB $(10 \mu \mathrm{M}, 25 \mu \mathrm{M}$, and $50 \mu \mathrm{M} ; 6 \mathrm{~h}$ ). (C) qRT-PCR of eRNA transcription levels in BET inhibitor-treated HepG2 cells $(5 \mu \mathrm{M}, 24 \mathrm{~h})$. The values are the mean \pm SD from triplicate well measurements. ${ }^{*}, p<0.05$ and $* *, p<0.01$.
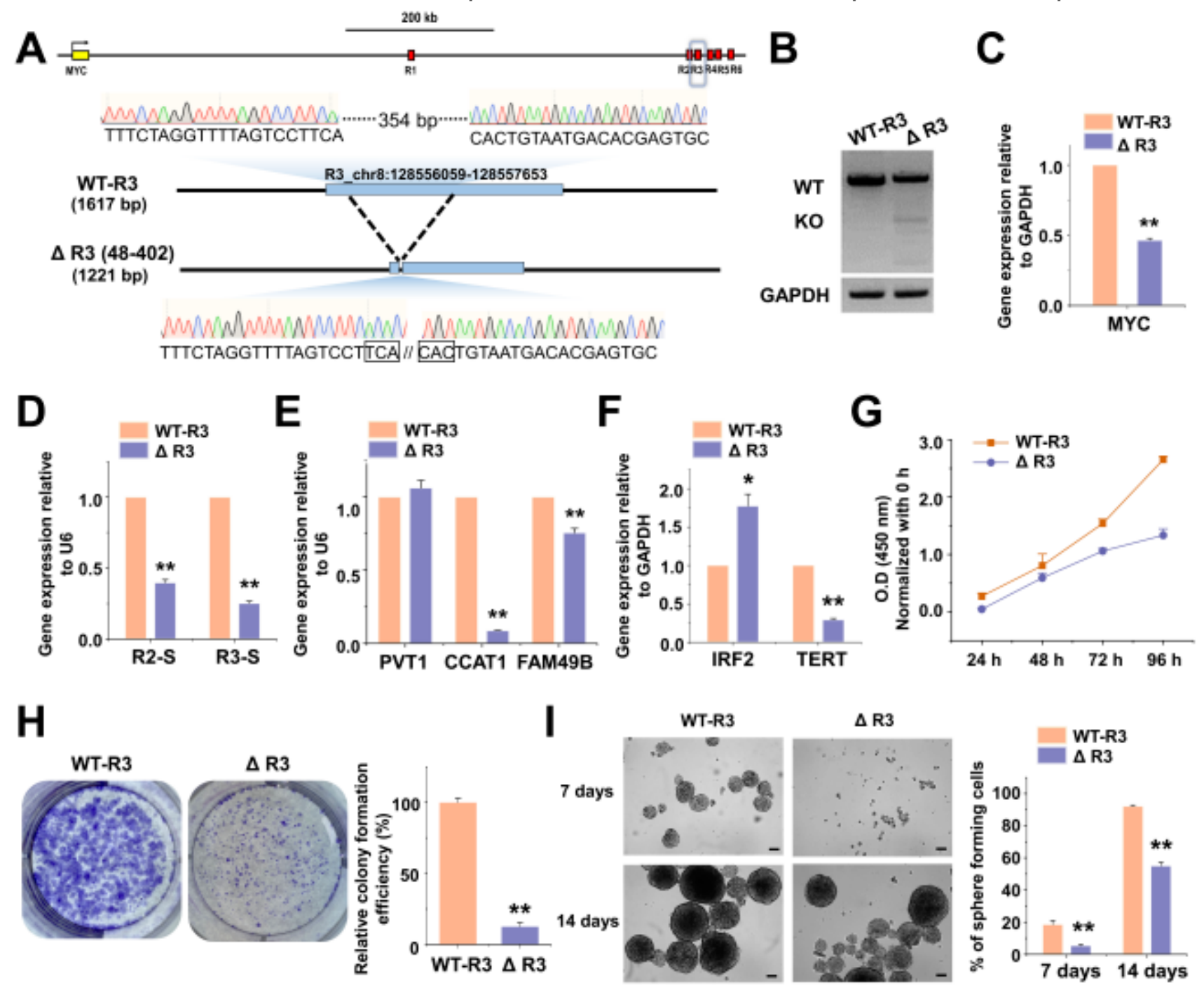

\section{Figure 4}

Generation of MYC enhancer region-deleted HCC cells via the CRISPR-Cas9 system (A) Schematic illustration of the MYC enhancer locus structure and wild-type and mutant allele sequences around the target locus. A modified allele sequence obtained from the R3-edited HCC cells is shown below. Detection of mutations introduced by gRNA-Cas9 targeting the R3 region via PCR genomic DNA from WT and R3edited HCC cells. (B) Agarose gel showing the genotyping PCR products amplified from the CRISPR-Cas9 targeted region. (C-F) qRT-PCR of MYC mRNA expression levels (C), eRNA transcription levels at R2 and R3 (D), and MYC-related gene mRNA expression levels (E, F) in WT and R3-edited HCC cells. The values are the mean \pm SD from triplicate well measurements. ${ }^{*}, p<0.05$ and $* *, p<0.01$. (G) Cell proliferation 
was determined using a WST-1 assay and represented by the relative absorbance at $450 \mathrm{~nm}$. WT and edited HCC cells were cultured in a growth medium for $96 \mathrm{~h}$. The obtained absorbance was normalized to each $0 \mathrm{~h}$ absorbance. The data represent three biologically independent experiments. $* *, p<0.01$. $(H)$ Colony formation ability of WT and R3-edited HCC cells. Cells were grown for 10 days and stained with crystal violet. The relative colony formation efficiency was measured as a percentage of the area covered by the colonies. The data represent three biologically independent experiments. $*$, $p<0.01$. (I) WT and R3-edited HCC cells were cultured in cancer stem cell (CSC) growth media for the sphere formation assay under ultralow adherence conditions. Cells grown for 7 days and 14 days in sphere-forming conditions are shown in bright-field images taken with a $4 \mathrm{X}$ objective. The number of large spheres (over $100 \mu \mathrm{m}$ ) was counted; scale bar $=100 \mu \mathrm{m}$. The data represent three biologically independent experiments. ${ }^{\star *}, \mathrm{p}<$ 0.01 . 
A


Figure 5

Effects of ASO treatment targeting MYC eRNA in HCC cells (A) Schematic illustration of the MYC enhancer locus structure and USCS genome browser view of the GRO-seq peak, H3K27ac enrichment, and p300 binding site along the R2 and R3 regions. Target ASOs were designed to bind to the R2 and R3 regions. (B, C) HCC cells were treated with nontargeting ASO (ASO NC) or an ASO targeting the knockdown of MYC eRNA (ASO-R2-P1, ASO_R3_P2, respectively). Relative quantitation of R2 (B) and R3 
(C) eRNA expression levels in ASO-treated HCC cells was conducted by qRT-PCR. (D-F). Relative quantitation of MYC mRNA expression levels (D) and MYC-related gene mRNA expression levels $(E, F)$ in ASO-treated Huh7 HCC cells was conducted by qRT-PCR. The values are the mean \pm SD from triplicate well measurements. ${ }^{*}, p<0.05$ and $* *, p<0.01$. (G) Cell proliferation was determined using a WST-1 assay and represented by the relative absorbance at $450 \mathrm{~nm}$. Absorbance was measured every $24 \mathrm{~h}$ from $0 \mathrm{~h}$ to $96 \mathrm{~h}$ after transfection of ASO. The data represent three biologically independent experiments. ${ }^{\star}, \mathrm{p}$ $<0.01$. (I) For the sphere formation assay, ASO-transfected HCC cells were cultured in a growth medium. Cells grown for 7 days and 14 days in sphere-forming conditions are shown in bright-field images taken with a 10X objective. The number of large spheres (over $100 \mu \mathrm{m}$ ) was counted; scale bar $=100 \mu \mathrm{m}$. The data represent three biologically independent experiments. ${ }^{* *}, p<0.01$.

\section{Supplementary Files}

This is a list of supplementary files associated with this preprint. Click to download.

- AdditionalfileMYC.docx 